\title{
Desenvolvimento e Avaliação das Características Psicométricas do Questionário de Auto-Percepção de Inteligência Emocional (QIE-AP)
}

\author{
Development and Evaluation of the Psychometric Properties of the Self-Perception \\ of Emotional Intelligence Questionnaire (EIQ-SP)
}

\author{
Andreia Pereira Teques ${ }^{*}, a$, Ginés Llorca-Ramón ${ }^{a}$, Glória Bueno-Carrera ${ }^{a}$, \\ José Pais-Ribeiro $^{b} \&$ Pedro Teques ${ }^{c}$ \\ ${ }^{a}$ Universidade de Salamanca, Salamanca, Espanha, ${ }^{b}$ Universidade do Porto, Porto, Portugal \\ $\&{ }^{c}$ Instituto Politécnico de Santarém, Santarém, Portugal
}

\begin{abstract}
Resumo
Este estudo pretendeu desenvolver e avaliar as características psicométricas de um novo instrumento para avaliar a autopercepção de capacidades de inteligência emocional (IE) baseado no modelo de IE de Mayer e Salovey (1997): o Questionário de Auto-Percepção de Inteligência Emocional (QIEAP). Foram desenvolvidos dois estudos transversais. A amostra inclui 401 participantes $(n 1=191$; $n 2=210$ ) com idades entre 16 e 75 anos. Os dados foram analisados considerando uma análise fatorial exploratória (AFE), análise fatorial confirmatória (AFC), análise multigrupos, confiabilidade, e análises de correlação. Os resultados da análise fatorial exploratória suportam a retenção de quatro fatores do modelo teórico original: percepção, avaliação e expressão emocional, facilitação emocional do pensamento, compreensão e análise emocional, e regulação emocional. A análise fatorial confirmatória demonstrou um bom ajustamento dos dados à estrutura original de quatro fatores, e os fatores revelaram confiabilidade, validade convergente e discriminante. Adicionalmente, as duas análises multigrupos demonstraram que o modelo final é totalmente invariante entre as duas amostras independentes e parcialmente invariantes entre gêneros. $\mathrm{O}$ estudo suporta a validade e confiabilidade inicial do QIE-AP, tornando-o num instrumento útil na área da IE.
\end{abstract}

Palavras-chave: Inteligência emocional, desenvolvimento psicométrico, avaliação.

\begin{abstract}
The purpose of this study was to develop and psychometrically evaluate a new measure of selfperception of emotional intelligence (EI) grounded on the Mayer and Salovey framework (1997): the Emotional Intelligence Self-Perception Questionnaire (EIQ-SP). Two cross-sectional studies with self-reported questionnaires were conducted. The total sample included 401 participants $(n 1=$ $191 ; n 2=210$ ) between 16 to 75 years old. Data were analyzed through exploratory factor analyses (EFA), confirmatory factor analyses (CFA), multi-group CFAs as well as reliability and correlational analyses. The results of an exploratory factor analysis supported the retention of 4 factors in the original measurement model: perceiving and expressing emotion, assimilating emotion in thought, understanding emotions, and reflectively regulating emotions. Confirmatory factor analyses supported the tenability of the 4-factor measurement model. All factors also show reliability, as well as convergent and discriminant validity. Additionally, two multiple-group CFAs showed that the final model was totally invariant between the two independent samples and partially invariant across gender samples. This study provided support for the initial reliability and validity of the EIQ-S and showed it as a useful instrument for research on emotional intelligence.

Keywords: Emotional intelligence, psychometric development, evaluation.
\end{abstract}

Nos últimos anos têm sido publicados vários trabalhos científicos sobre a Inteligência Emocional (IE). Acompanhando o interesse pela IE, foram desenvolvidos estudos

\footnotetext{
* Endereço para correspondência: Universidade de Salamanca, Faculdade de Medicina, Campus Miguel de Unamuno, Calle Alfonso X El Sabio, s/n, Salamanca, Espanha 37007. E-mail: apsousapereira@gmail.com
}

em várias áreas, principalmente na área educacional, organizacional e saúde mental (Mayer, Salovey, Caruso, \& Sitarenios, 2003). Neste sentido, múltiplos estudos de revisão sobre IE evidenciam a sua relação positiva com componentes intra e inter pessoais, sociais, profissionais, e de saúde, essenciais para o bem-estar global (Bhullar, Schutte, \& Malouff, 2012; Goldenberg, Matheson, \& Mantler, 2006; Limonero, Sábado, \& Fernández-Castro, 
Teques, A. P., Llorca-Ramón, G., Bueno-Carrera, G., Pais-Ribeiro, J. \& Teques, P. (2015). Desenvolvimento e Avaliação das Características Psicométricas do Questionário de Auto-Percepção de Inteligência Emocional (QIE-AP).

2006; Mayer, 2006; Mayer, Roberts, \& Barsade, 2008; Schutte, Malouff, \& Bhullar, 2007; Schutte, Malouff, Simunek, McKenley, \& Hollander, 2002; Schutte et al., 2010; Veríssimo, 2005).

No entanto, apesar do constante desenvolvimento de estudos considerando a IE, uma das limitações destes estudos são a sua incoerência teórica ou simplesmente ausência de qualquer modelo teórico como referência (Pérez-González, Petrides, \& Furnham, 2007). Neste âmbito, vários autores descrevem a IE como uma mistura eclética de características, tais como a felicidade, a autoestima, o otimismo, e a auto-realização, mais do que uma habilidade (Bar-On, 2004; Petrides \& Furnham, 2001). De acordo com Bedwell (2003), perante as diferentes teorias sobre IE, surgiram como consequência, conceitos e instrumentos de medida diferentes. Nesta amplitude teórica, Mayer, Caruso e Salovey (2000; Mayer, Salovey, \& Caruso 2002), destacam a distinção teórica entre os modelos baseados nos traços de personalidade ou descritos como modelos mistos (e.g., Bar-On, 1997; Goleman, 1995/2003), identificando-se a IE como traço (Petrides \& Furnham, 2001) e os modelos baseados nas capacidades cognitivas (e.g., Mayer \& Salovey, 1997), definindo e medindo a IE como uma capacidade (Petrides \& Furnham, 2001). Neste ponto surge a crítica aos modelos de traço ou mistos, como, por exemplo, ao revelarem pouca credibilidade na possibilidade de definir IE. A este nível os autores do nosso modelo teórico de referência, Modelo de Inteligência Emocional de Mayer e Salovey (1997), definem IE como: a capacidade para sentir e avaliar a emoção com exatidão, a capacidade para identificar e/ou criar sentimentos quando estes facilitam o pensamento, a capacidade para compreender a emoção e o conhecimento emocional, e a capacidade para regular as emoções que incentivam o crescimento emocional e intelectual. (p. 13)

O Modelo de Inteligência Emocional de Mayer e Salovey (1997), tem sido apontado como o modelo mais plausível para avaliar e compreender a IE, quer em termos de definição e métodos de medida (Extremera \& Fernández-Berrocal, 2005; Mayer et al., 2000; Roberts, Zeidner, \& Matthews, 2001), bem como, pela sua teoria não se basear em promessas insustentáveis em relação ao potencial da IE (Woyciekoski \& Hutz, 2009). O modelo é constituído por quatro dimensões que abrangem processos psicológicos dos mais básicos aos mais elevados. $\mathrm{Na}$ base, apresenta-se a Percepção, Avaliação e Expressão da Emoção, onde os indivíduos identificam as emoções e o seu conteúdo emocional. O segundo nível da Facilitação Emocional do Pensamento refere-se à ação da emoção sobre a inteligência, este descreve os eventos emocionais que assistem ao processo intelectual. A terceira dimensão relaciona-se com a capacidade de Compreensão e Análise das Emoções utilizando o Conhecimento Emocional, focando a descrição e a relação entre as emoções, e reconhecendo as semelhanças e as diferenças das mesmas e os significados destas nas relações. No topo do modelo encontra-se a dimensão Regulação Reflexiva das Emoções para Promover o Crescimento Pessoal e Intelectual, definindo-se como a regulação consciente das emoções, fomentando o crescimento emocional e intelectual (Mayer \& Salovey, 1997). Em termos psicométricos foram desenvolvidos vários instrumentos para avaliar a IE, considerando o Modelo de Inteligência Emocional de Mayer e Salovey (1997). Usualmente, a avaliação da IE como capacidade recorre ao instrumento de performance, Mayer-Salovey-Caruso Emotional Intelligence Test (MSCEIT, Mayer et al., 2002), que tem revelado boas características psicométricas. Por outro lado, os testes de auto-relato mais utilizados são o Emotional Quotient Inventory de Bar-On (EQ-I, 1997) que apresenta uma relação entre vários construtos, suscitando alguma confusão teórica, e o Self-Report EI Test de Schutte et al. (SREIT, 1998). Todavia, o SREIT apesar de apresentar uma boa consistência interna e uma boa confiabilidade teste-reteste, alguns autores criticaram as suas propriedades psicométricas devido a não se revelar unidimensional, conforme o defendido por Schutte (Brackett \& Mayer, 2003). Da mesma forma, Teques (2009) realizou a tentativa de adaptar o SREIT à população portuguesa $\mathrm{e}$ os resultados foram similares, apresentando bons índices de confiabilidade através do alpha de Cronbach, mas a análise fatorial exploratória revelou uma estrutura de 11 fatores, contradizendo o modelo de base de Mayer e Salovey (1997).

Em Portugal, foram realizados estudos de adaptação dos seguintes instrumentos de IE: o Trait Meta-Mood Scale de Salovey, Mayer, Goldman, Turvey, e Palfai (1995) foi adaptado por Queirós, Fernández-Berrocal, Extremera, Carral, e Queirós (2005) numa versão modificada, sendo igualmente composta por apenas três dimensões tal como a escala original, não remetendo as quatro do modelo de base; o Emotional Skills and Competence Questionnaire de Taksic, Takalcic, e Brajkovic (2001), foi outro instrumento adaptado à população portuguesa por Lima-Santos e Faria (2005) baseado no modelo de Mayer e Salovey (1997), apresenta apenas 3 das dimensões que o compõe e nem todas apresentam uma consistência interna satisfatória. $\mathrm{E}$ a Multi-factor Emotional Intelligence Scale de Mayer et al. (2002) foi adaptada por Franco (2003) apenas junto a uma população do $1^{\circ}$ ciclo, parecendo existir ainda muito para desenvolver a nível de instrumentos de desempenho da IE (Franco, 2007).

Neste âmbito, na construção do instrumento teve-se em conta por um lado, as recomendações de Franco (2007) e Rodrigues, Rebelo, e Coelho (2011), para se adaptar ou desenvolver escalas de IE com boas características psicométricas, visto as escalas adaptadas à população portuguesa não revelarem consistência interna satisfatória, ou estas não se ajustarem ao modelo de base, como os dados descritos anteriormente. Assim como, por outro lado, respeitou-se integramente o modelo teórico de base, sem acrescentar outros construtos na sua composição multidimensional ou retirar qualquer dimensão que o compõe, conforme se assinalou em outras escalas de auto-percepção de IE, o que leva a uma designação ou 
descrição insuficiente no domínio da IE (e.g. TMMS, EQI, SREIT; Pérez, Petrides, \& Furnham, 2005). O formato do instrumento é de auto-relato ao invés de tarefas de performance defendidas pelos autores de referência, tal porque, apesar dos testes de performance e/ou de IE como capacidade existirem há muito tempo, continuam a existir dúvidas relativamente à consistência interna e à estrutura fatorial. Em relação às medidas de IE como traço e/ou de auto-relato, poucas são aquelas que se desenvolveram com um marco teórico claro, assim como não apresentam fundamentos empíricos consistentes (Pérez-González et al., 2007), destacando-se o nosso instrumento de outras escalas de auto-percepção da IE visto manter-se as quatro dimensões do Modelo de referência, não apresentando sobreposições de escalas de construtos existentes e por se distinguir o que é unicamente construto da IE dos traços de personalidade (Petrides \& Furnham, 2001). Selecionamos o formato de auto-relato na medida em que para além dos benefícios práticos da sua aplicação, desta forma conseguiremos aceder, conforme Gardner (1993/1995), à inteligência pessoal na dimensão mais intrapessoal, em que o sujeito revela a sua capacidade de auto-conhecimento, através do qual orienta o seu comportamento. Zeidner, Shani-Zinovich, Matthews e Roberts, (2005) acrescentam que, avaliar a auto-percepção de IE se revela importante na área da inteligência tradicional.

Deste modo, o propósito do presente estudo foi construir e validar um questionário baseado no modelo teórico de Mayer e Salovey (1997) para avaliar a auto-percepção das capacidades da IE. Operacionalizando-se a investigação em dois estudos, cujo objetivo geral é avaliar as propriedades psicométricas do Questionário de Auto-Percepção de Inteligência Emocional (QIE-AP). O primeiro estudo pretende explorar a estrutura e composição fatorial do QIE-AP através de uma análise fatorial exploratória, e o segundo estudo, visa a verificação da validade de construto, por meio de uma análise fatorial confirmatória.

\section{Estudo 1}

O propósito do Estudo 1 foi explorar a composição fatorial e a estrutura do QIE-AP para determinar o grau de correspondência da série de itens iniciais com as dimensões do modelo de Mayer e Salovey (1997), correspondentes aos fatores de Percepção, avaliação e expressão emocional, Facilitação emocional do pensamento, Compreensão e análise emocional, e Regulação emocional.

\section{Método do Estudo 1}

\section{Amostra e Procedimentos}

Os participantes do estudo foram 191 cidadãos portugueses que habitam no norte e centro de Portugal (64,4\% mulheres; $35,6 \%$ homens), com idades compreendidas entre os 16 e os 75 anos ( $M^{\text {idade }}=31$ anos). A maioria da amostra é solteira (52,4\%), estudante $(27,2 \%)$, e com o Ensino Secundário (27,2\%).
Respeitando as indicações do comitê de ética da universidade, os participantes foram previamente esclarecidos sobre os objetivos e procedimentos da investigação, assumindo a confidencialidade dos dados, e todos os participantes facultaram o seu consentimento informado previamente à aplicação dos instrumentos. Os critérios de inclusão para a amostra foram: apresentar nacionalidade e domínio da língua portuguesa, idade compreendida igual ou superior a 16 anos, saber ler e escrever e não padecer de doença crônica.

\section{Instrumentos}

Os instrumentos incluíram um questionário sóciodemográfico e uma versão preliminar do Questionário de Auto-Percepção de Inteligência Emocional (QIE-AP). O Questionário sócio-demográfico especificou as variáveis sexo, idade, profissão, estado civil, escolaridade e município onde habita.

Construção do Instrumento de Medida. A construção da versão preliminar do QIE-AP baseada no Modelo de Inteligência Emocional de Mayer e Salovey (1997), foi executada considerando um processo de três etapas. $\mathrm{Na}$ primeira etapa, foram criados um conjunto de itens com vistas a avaliar cada um dos construtos do modelo teórico. Na segunda etapa, foi avaliada a validade de conteúdo. Neste âmbito, a versão preliminar do questionário foi analisada por um grupo de quatro especialistas com o objetivo de analisar a relevância teórica de cada item para cada construto. Os critérios de inclusão dos especialistas foram a sua experiência reconhecida no desenvolvimento de investigação e prática na área da psicologia clínica e da saúde, e a sua experiência no desenvolvimento de instrumentos psicométricos. Na última etapa, foi criado um grupo focado com oito pessoas com idades entre os $20 \mathrm{e}$ os 53 anos, com o intuito de avaliar a clareza, terminologia, compreensão e formato dos itens. Após análise, o formato de alguns itens foram ligeiramente modificados sem alterar a sua correspondência conceitual. A versão preliminar do questionário utilizada no presente estudo teve no total 30 itens, subdivididos em quatro escalas correspondentes às dimensões do modelo teórico de Mayer e Salovey (1997). Os itens foram construídos sob a forma de afirmação, optando-se pelo uso de uma escala tipo Likert de cinco alternativas (1 - Discordo totalmente a 5 - Concordo totalmente).

\section{Análise dos Dados}

Procedeu-se à análise dos dados através do programa de tratamento estatístico Statistical Package for Social Sciences (SPSS), versão 20.0 para Windows. A estrutura fatorial do QIE-AP foi examinada inicialmente através da análise fatorial exploratória (AFE), utilizando o método de extração dos componentes principais, seguido da rotação oblíqua dos fatores, para que se possa obter uma solução fatorial mais clara e objetiva, maximizando assim os pesos fatoriais dos itens (Brown, 2006). A análise dos dados seguiu seis etapas fundamentais: (a) Critério 
Teques, A. P., Llorca-Ramón, G., Bueno-Carrera, G., Pais-Ribeiro, J. \& Teques, P. (2015). Desenvolvimento e Avaliação das Características Psicométricas do Questionário de Auto-Percepção de Inteligência Emocional (QIE-AP).

de Kaiser-Meyer-Olkin (KMO): reter fatores com valor próprio igual ou superior a 1; (b) Peso fatorial: reter itens com pesos iguais ou superiores a 0,3 ; (c) Pesos fatoriais cruzados: inexistência de itens com pesos fatoriais relevantes $(>0,30)$ em mais do que um fator. Caso aconteça e a diferença entre eles não for superior 0,15 , o item deve ser eliminado; (d) A percentagem da variância: o conjunto dos fatores retidos deve explicar pelo menos $40 \%$ da variância dos resultados; (e) Consistência interna: a confiabilidade interna do fator deve ser igual ou superior a 0,70 (Nunnaly \& Bernstein, 1994); e (f) só devem ser retidos os fatores com pelo menos três itens.

\section{Resultados}

A medida de adequação da amostra (teste $\mathrm{KMO}=$ $0,83)$ indicou que a análise dos componentes principais pode ser realizada e o teste de esfericidade significativo $(2701,002 ; p<0,05)$ revelou que os itens são interdependentes (Worthington \& Whittaker, 2006). Assim, verificou-se uma solução de quatro fatores que explicam $51,32 \%$ do total da variância dos resultados. Na Tabela 1 apenas são indicados os pesos fatoriais correspondentes à matriz de estrutura que têm em consideração a correlação entre os fatores, bem como aqueles que são considerados relevantes como valor mínimo para poderem ser interpretados $(0,30$; Hair, Black, Babin, \& Anderson, 2010). Neste sentido, os pesos fatoriais variam entre 0,49 e 0,83 e os coeficientes de consistência interna do $\alpha$ de Cronbach são aceitáveis (Nunnaly \& Bernstein, 1994): 0,73 (Fator 1: Percepção, avaliação e expressão emocional), 0,70 (Fator 2: Facilitação emocional do pensamento), 0,73 (Fator 3: Compreensão e análise emocional), e 0,77 (Fator 4: Regulação emocional). A versão final da escala inclui 18 itens.

Tabela 1

Médias, Desvios-Padrão, Comunalidades, e a Solução de Quatro Fatores do QIE-AP (Rotação oblíqua)

\begin{tabular}{|c|c|c|c|c|c|c|}
\hline Itens & $M \pm D P$ & Comunalidades & Fator 1 & Fator 2 & Fator 3 & Fator 4 \\
\hline Item 23 & $4,01 \pm 0,85$ & 0,58 & 0,73 & & & \\
\hline Item 16 & $3,97 \pm 0,91$ & 0,51 & 0,67 & & & \\
\hline Item 29 & $3,83 \pm 0,90$ & 0,45 & 0,65 & & & \\
\hline Item 3 & $4,67 \pm 0,63$ & 0,41 & 0,60 & & & \\
\hline Item 10 & $4,14 \pm 0,88$ & 0,37 & 0,50 & & & \\
\hline Item 20 & $4,25 \pm 0,78$ & 0,41 & 0,49 & & & \\
\hline Item 18 & $3,87 \pm 0,97$ & 0,60 & & 0,76 & & \\
\hline Item 14 & $4,07 \pm 0,85$ & 0,59 & & 0,73 & & \\
\hline Item 9 & $3,69 \pm 0,89$ & 0,54 & & 0,72 & & \\
\hline Item 30 & $4,08 \pm 0,93$ & 0,45 & & 0,64 & & \\
\hline Item 4 & $4,55 \pm 0,90$ & 0,54 & & & 0,71 & \\
\hline Item 6 & $4,13 \pm 0,97$ & 0,52 & & & 0,67 & \\
\hline Item 12 & $4,30 \pm 0,83$ & 0,57 & & & 0,06 & \\
\hline Item 26 & $4,09 \pm 1,00$ & 0,42 & & & 0,02 & \\
\hline Item 2 & $4,33 \pm 0,83$ & 0,39 & & & 0,52 & \\
\hline Item 19 & $4,14 \pm 0,91$ & 0,71 & & & & 0,83 \\
\hline Item 21 & $4,05 \pm 0,84$ & 0,67 & & & & 0,80 \\
\hline Item 24 & $4,34 \pm 0,85$ & 0,60 & & & & 0,75 \\
\hline Variância (\%) & & & 14,06 & 12,88 & 12,86 & 12,50 \\
\hline$\alpha$ Cronbach & & & 0,73 & 0,70 & 0,73 & 0,77 \\
\hline
\end{tabular}




\section{Sumário do Estudo 1}

O objetivo do Estudo 1 foi explorar a dimensionalidade latente e a estrutura dos itens do QIE-AP, e analisar as inter-relações entre os fatores. Os resultados indicam que os 18 itens do QIE-AP exibem uma estrutura simples interpretável, sendo consistente com o Modelo de Inteligência Emocional (Mayer \& Salovey, 1997). Acrescenta-se ainda, que a estrutura psicométrica do QIE-AP revelou confiabilidade.

\section{Estudo 2}

O propósito do Estudo 2 foi ampliar a validade do construto das pontuações do QIE-AP, testando o ajustamento do modelo identificado no Estudo 1, executando uma análise fatorial confirmatória (AFC) com uma amostra independente. Adicionalmente, foram efetuadas duas análises multigrupos para determinar a invariância das pontuações do QIE-AP entre as amostras independentes do presente Estudo 2 e do Estudo 1, e entre os gêneros. A invariância refere-se ao significado equivalente das pontuações em grupos diferentes (Cheung \& Rensvold, 2002), sendo as comparações entre grupos de base uma consideração importante no desenvolvimento de escalas de medida (Hoyle \& Smith, 1994).

\section{Método do Estudo 2}

\section{Amostra}

A amostra é constituída por 210 participantes (73,3\% mulheres; $26,7 \%$ homens), com idades compreendidas entre os 16 e os 71 anos (Midade $=33$ anos). Na sua maioria eram estudantes $(40,5 \%)$, solteiros $(55,2 \%)$, e com o Ensino Secundário completo (28,6\%). Os procedimentos na coleta dos dados do Estudo 2 foram idênticos aos do Estudo 1.

\section{Instrumentos}

Os instrumentos utilizados foram o Questionário sociodemográfico e a versão final do QIE-AP com os 18 itens, resultantes da análise fatorial exploratória realizada no Estudo 1. O QIE-AP é constituído por quatro dimensões consistentes com o Modelo de Inteligência Emocional de Mayer e Salovey (1997): (a) percepção, avaliação e expressão emocional, que visa avaliar a capacidade da pessoa descrever e distinguir as suas emoções e as das outras pessoas. A escala é composta por quatro itens (e. g., "Através do tom de voz e dos gestos dos outros, consigo perceber se estão tristes ou zangados"); (b) facilitação emocional do pensamento, pretende compreender se os sujeitos apoiam o seu processo de informação e decisão nas emoções sentidas. Esta escala é constituída por cinco itens (e. g., "Fico feliz por pensar nas coisas boas que tenho"; (c) compreensão e análise emocional. Orienta-se para a facilidade com que a pessoa associa as emoções a situações e pessoas específicas. A escala é avaliada por seis itens (e. g., "A perda de alguém de quem gosto deixa- me triste"; (d) regulação emocional, relaciona-se com a capacidade da pessoa controlar a sua postura, expressão e decisão comportamental perante emoções positivas ou negativas, levando à ampliação das situações prazerosas e ao evitamento das situações que despoletam emoções negativas, sem prejudicar o sucesso pessoal e social, conotando sempre uma aprendizagem e crescimento emocional favorecendo o seu bem-estar, bem como o dos demais. A regulação emocional é avaliada considerando três itens (e. g., "Procuro fazer o que me dá mais prazer"). Todos os itens são respondidos pelos participantes através de uma escala Likert de cinco pontos $(1-$ Discordo totalmente a 5 - Concordo totalmente).

\section{Análise dos Dados}

A análise de dados realizou-se em cinco etapas. Em primeiro lugar, os dados foram analisados para identificar potenciais valores discrepantes, missings ou discrepâncias que influenciem de forma negativa as análises subsequentes. Em segundo, foram calculadas as estatísticas descritivas dos itens do QIE-AP para selecionar o procedimento estatístico apropriado para a análise fatorial confirmatória (AFC). Em terceiro, foi executada a AFC recorrendo ao AMOS 20 (SPSS Inc, Chicago IL) para avaliar o ajustamento do modelo de quatro fatores do QIE-AP extraído dos resultados oriundos do Estudo 1. Neste âmbito, o ajustamento do modelo foi avaliado por um conjunto de índices de ajustamento. Especificamente, um bom ajustamento foi considerado quando o qui-quadrado $\left(\chi^{2}\right)$ e os seus graus de liberdade são inferiores a 3,0, e o comparative-of-fitindex (CFI) e o Tucker-Lewis Index (TLI) foram superiores a 0,90 (Hair et al., 2010). Valores do root mean square error of approximation (RMSEA) abaixo de 0,06 são indicadores de bom ajustamento, enquanto que se forem entre 0,08 e 0,10 são considerados aceitáveis (Byrne, 2000). Em quarto lugar foram analisados os princípios de confiabilidade, validade convergente e discriminante. A confiabilidade dos fatores foi estimada através do coeficiente do $\alpha$ Cronbach, sendo que valores acima de 0,70 foram considerados aceitáveis (Nunnaly \& Bernstein, 1994). A variância extraída média (VEM) foi avaliada como critério de validade convergente e valores superiores a 0,50 foram considerados adequados para demonstrar validade convergente (Fornell \& Larcker, 1981; Hair et al., 2010). A validade discriminante foi assumida quando a VEM de cada fator é superior ao quadrado das correlações entre os outros fatores (Fornell \& Larcker, 1981).

Em último lugar, foram executadas duas análises multigrupos com o objetivo de testar a invariância do modelo entre as amostras do Estudo 1 e do presente estudo, e a invariância das respostas entre gêneros. A invariância entre modelos foi analisada segundo os valores do teste da significância do qui-quadrado e da diferença dos valores do CFI ( $\triangle$ CFI; Cheung \& Rensvold, 2002). Se os valores do $\chi^{2}$ na comparação entre modelos não for estatisticamente significativo $(p>0,05)$, a hipótese de invariância deve ser considerada. Contudo, os autores referem que o teste do 
Teques, A. P., Llorca-Ramón, G., Bueno-Carrera, G., Pais-Ribeiro, J. \& Teques, P. (2015). Desenvolvimento e Avaliação das Características Psicométricas do Questionário de Auto-Percepção de Inteligência Emocional (QIE-AP).

qui-quadrado é influenciado pelo tamanho da amostra. Neste sentido, propõem a análise às alterações no $\Delta \mathrm{CFI}$ superiores a 0,01, como um critério alternativo para avaliar a invariância dos modelos. A avaliação da invariância entre grupos é executada pela testagem sequencial dos seguintes modelos: modelo 1 - não constrito; modelo 2 - pesos fatoriais; modelo 3 - variâncias-covariâncias.

\section{Resultados}

\section{Análises Preliminares}

Foi analisada a normalidade dos dados e a existência de missings (Tabachnick \& Fidell, 2007). Não foram encontradas missings, nem foram identificados outliers, tanto univariados (valores de skewness $<3$; valores de kurtosis $<7$; Kline, 2011) como multivariados [distância Mahalanobis $\left.<\chi^{2}(3)=40,236\right]$

Tabela 2

Pesos Fatoriais e Coeficientes de Consistência Interna ( $\alpha$ Cronbach)

Fatores/ Pesos fatoriais

Itens

Percepção, avaliação e expressão emocional

5. Existem pessoas que se fazem parecer mais felizes do que realmente são.

8. Através do tom de voz e dos gestos dos outros, consigo perceber

0,69

0,73

se estão tristes ou zangados.

0,77

0,68

10. Algumas pessoas conseguem fingir estar tristes para chamar a atenção.

0,69

18. Os animais de estimação podem fazer-nos sentir bem.

Facilitação emocional do pensamento

1. Fico feliz por pensar nas coisas boas que tenho.

3. Quando me sinto feliz sinto-me com menos energia. (-)

0,63

0,68

4. Quando estou mais triste fico com menos vontade de fazer coisas.

0,64

7. Quando estou alegre faço mais coisas.

0,73

16. Quando estou triste tenho pensamentos mais positivos. (-)

0,51

Compreensão e análise emocional

2. A perda de alguém de quem gosto deixa-me triste

6. Existem pessoas que gosto e outras que me são indiferentes.

0,67

0,66

9. Num mau momento pode-se sentir várias emoções como medo, tristeza, raiva. $\quad 0,70$

12. Quando me elogiam sinto alegria e orgulho ao mesmo tempo.

0,65

14. Os pensamentos negativos deixam-me triste ou desamimado (a).

0,45

17. Quando tenho em conta os meus sentimentos é mais fácil tomar decisões.

0,84

Regulação emocional

0,77
11. Procuro fazer o que me dá mais prazer.
0,74
13. Tento fazer atividades que eu e os outros gostamos.
0,74
15. Convivo o mais que posso com as pessoas que me fazem sentir bem.
0,52

$\alpha$ Cronbach

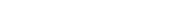


pensamento $(0,41)$ e à Compreensão e análise emocional $(0,38)$ revelaram ser insuficientes para garantir validade convergente. No entanto, a avaliar pela matriz de correlação, verifica-se que os quatro fatores correlacionam-se entre eles. Por sua vez, todos os fatores revelaram validade discriminante, visto que nenhum quadrado das correlações excedeu os valores da VEM associados a cada fator (ver Tabela 3). Considerando a originalidade dos fundamentos teóricos da escala, não foi executada qualquer re-especificação à estrutura do modelo.

Tabela 3

Correlações, Médias, Desvios-Padrão, e Variância Extraída Média

\begin{tabular}{lcccc}
\hline \multirow{2}{*}{ Fatores } & \multicolumn{5}{c}{ Matriz de correlação } \\
\cline { 2 - 5 } & 1 & 2 & 3 & 4 \\
\hline Percepção & 1 & & & \\
Facilitação & $0,27^{*}$ & 1 & & \\
Compreensão & $0,30^{*}$ & $0,42^{*}$ & 1 & \\
Regulação & $0,16^{*}$ & $0,23^{*}$ & $0,22^{*}$ & 1 \\
$M$ & 3,94 & 4,21 & 4,09 & 4,10 \\
$D P$ & 0,67 & 0,69 & 0,59 & 0,82 \\
VEM & 0,51 & 0,41 & 0,38 & 0,61 \\
\hline
\end{tabular}

Nota. O quadrado da correlação mais elevada é inferior aos valores da VEM, revelando validade discriminante em todos os fatores.

$* p<0,01$.

\section{Invariância do Modelo}

Na Tabela 4 encontram-se os resultados das duas análises multigrupos para testar a invariância do modelo nos momentos independentes de avaliação utilizando as amostras do Estudo $1(n=191)$ e do presente estudo $(n=$ $210)$, e nas respostas entre mulheres $(n=277)$ e homens $(n=124)$. Primeiro, a análise multigrupos executada com as duas amostras independentes revelou bons índices de ajustamento do modelo não constrito [modelo $1: \chi^{2}$ (258) $=392,62(p=0,001)$, TLI $=0,90$, CFI $=0,93$, RMSEA $=0,03]$. Os modelos com os pesos fatoriais constritos [modelo 2: $\chi^{2}(\mathbf{2 7 2})=404,39(p=0,001), \mathrm{TLI}=0,90, \mathrm{CFI}$ $=0,94$, RMSEA $=0,03]$ e com as variâncias-covariâncias constritas revelaram ajustamentos adequados [modelo 3: $\chi^{2}(\mathbf{2 8 2})=423,82(p=0,001), \mathrm{TLI}=0,89, \mathrm{CFI}=0,93$, RMSEA $=0,03]$. $O$ teste de significância do qui-quadrado $\left(\chi^{2}\right)$ e das diferenças do CFI ( $\left.\triangle \mathrm{CFI}\right)$ não demonstraram alterações significativas quando comparados os modelos 1 e $2\left[\chi^{2 \text { dif }}(14)=11,77 ; p=0,62 ; \Delta \mathrm{CFI} \leq 0,01\right]$, bem como os modelos 1 e 3 [ $\left.\chi^{\text {2dif }}(24)=31,20 ; p=0,14 ; \Delta \mathrm{CFI} \leq 0,01\right]$.

Segundo, a análise multigrupos relativa à sensibilidade das respostas entre gêneros, o modelo constrito revelou valores ajustados [modelo $1: \chi^{2}(\mathbf{2 5 8})=373,48(p=0,001)$, $\mathrm{TLI}=0,90, \mathrm{CFI}=0,94, \mathrm{RMSEA}=0,03]$, bem como para os modelos com pesos fatoriais constritos [modelo $2: \chi^{2}(272)$ $=395,08(p=0,001), \mathrm{TLI}=0,90, \mathrm{CFI}=0,94$, RMSEA $=0,03]$ e variâncias-covariâncias constritas [modelo 3 : $\chi^{2}(282)=443,99(p=0,001)$, TLI $=0,88$, CFI $=0,92$, RMSEA $=0,03]$. O teste de significância do qui-quadrado $\left(\chi^{2}\right)$ e das diferenças do CFI ( $\left.\triangle \mathrm{CFI}\right)$ não demonstraram alterações significativas quando comparados os modelos 1 e $2\left[\chi^{2 \text { dif }}(14)=21,59 ; p=0,08 ; \Delta \mathrm{CFI} \leq 0,01\right]$. No entanto, foram encontradas diferenças significativas na comparação dos modelos 1 e 3 , tanto a nível do qui-quadrado [ $\chi^{\text {2dif }}(24)$ $=70,50 ; p=0,001]$, como do CFI $[\Delta \mathrm{CFI} \geq 0,01]$.

Tabela 4

Análises Multigrupo às Amostras dos Estudo $1(n=191)$ e Estudo $2(n=210)$, e entre Gêneros $(n=401)$

\begin{tabular}{|c|c|c|c|c|c|c|c|}
\hline Modelos & $\chi^{2}$ & $g l$ & $p$ & CFI & $\Delta \mathrm{CFI}$ & TLI & $\operatorname{RMSEA}\left(\mathrm{IC}_{90 \%}\right)$ \\
\hline \multicolumn{8}{|l|}{ Sub-estudo 1 vs Sub-estudo 2} \\
\hline M1 - Não constrito & 392,62 & 258 & 0,001 & 0,93 & - & 0,90 & $0,03(0,02,0,04)$ \\
\hline M2 - Pesos fatoriais & 404,39 & 272 & 0,285 & 0,94 & $\leq 0,01$ & 0,90 & $0,03(0,02,0,04)$ \\
\hline M3 - Variâncias-covariâncias & 423,82 & 282 & 0,172 & 0,93 & $\leq 0,01$ & 0,89 & $0,03(0,02,0,04)$ \\
\hline \multicolumn{8}{|l|}{ Mulheres vs Homens } \\
\hline M1 - Não constrito & 373,48 & 258 & 0,001 & 0,95 & - & 0,90 & $0,03(0,02,0,04)$ \\
\hline M2 - Pesos fatoriais & 395,08 & 272 & 0,087 & 0,95 & $\leq 0,01$ & 0,90 & $0,03(0,02,0,04)$ \\
\hline M3 - Variâncias-covariâncias & 443,99 & 282 & 0,001 & 0,94 & $\geq 0,01$ & 0,88 & $0,03(0,03,0,04)$ \\
\hline
\end{tabular}

\section{Sumário do Estudo 2}

O propósito do Estudo 2 foi avaliar a validade de construto dos resultados do QIE-AP através da confirmação do ajustamento do modelo de medida derivado do Estudo
1. Os resultados da AFC corroboram o ajustamento da solução de quatro fatores do modelo de medida do QIEAP. Cada um dos fatores revelou consistência interna aceitável, corroborando também com os valores atingidos 
Teques, A. P., Llorca-Ramón, G., Bueno-Carrera, G., Pais-Ribeiro, J. \& Teques, P. (2015). Desenvolvimento e Avaliação das Características Psicométricas do Questionário de Auto-Percepção de Inteligência Emocional (QIE-AP).

no Estudo 1. Adicionalmente, os resultados sugerem que os fatores da Percepção, avaliação e expressão emocional e Regulação emocional apresentam validade convergente. No entanto, os fatores de Compreensão e análise emocional e Facilitação emocional do pensamento revelaram valores insuficientes em relação à validade convergente. Por sua vez, os quatro fatores apresentaram valores que suportam a evidência de validade discriminante. Por último, o modelo de medida do QIE-AP revelou ser invariante nas duas amostras independentes, tanto a nível dos seus pesos fatoriais como das variâncias-covariâncias, sendo interpretado como indicador de validade cruzada (Loehlin, 2004). Em relação à invariância entre homens e mulheres, o modelo revelou ser parcialmente invariante, sugerindo invariância nos pesos fatoriais, mas variando entre as variâncias-covariâncias.

\section{Discussão}

O propósito geral do presente estudo foi analisar as propriedades psicométricas do QIE-AP na população portuguesa. Os resultados dos Estudos 1 e 2 revelaram que a estrutura dos fatores e a composição do QIE-AP reflete os quatro fatores do Modelo de Inteligência Emocional de Mayer e Salovey (1997), e sugerem que o QIE-AP é uma medida através da qual cada item mede um único construto latente (Kline, 2011). Por conseguinte, os resultados da análise fatorial confirmatória atestaram um bom ajustamento dos dados, revelando uma estrutura de quatro fatores delineada para aceder à auto-percepção das capacidades de inteligência emocional (IE), sendo consistente com o modelo teórico de base (Mayer \& Salovey, 1997).

Combinando a evidência de validade estrutural das pontuações do QIE-AP, a primeira análise multigrupos suporta a invariância das pontuações do QIE-AP entre as duas amostras, existindo evidência de validade cruzada (Loehlin, 2004). A segunda análise multigrupos demonstrou invariância parcial entre gêneros, dando suporte inicial para o uso do QIE-AP em homens e mulheres. A descrição da invariância nesta fase do desenvolvimento do QIE-AP é positiva, permitindo as comparações nos grupos de base entre homens e mulheres, sem necessidade de diferenciar futuramente os itens entre os gêneros.

Noutro âmbito, todos os fatores do QIE-AP parecem ser fidedignos (Nunnaly \& Bernstein, 1994) e revelaram validade discriminante, visto que a VEM de cada fator foi superior ao quadrado das correlações entre os outros fatores (Tabachnick \& Fidell, 2007). Contudo, os valores da VEM referentes aos fatores da Facilitação emocional do pensamento e da Compreensão e análise emocional revelaram ser insuficientes para garantir validade convergente $(<$ 0,50; Fornell \& Larcker, 1981). Em geral, estes resultados que consideram o critério da VEM para avaliar a validade convergente e discriminante, sugerem que os quatro fatores do QIE-AP são mutuamente exclusivos, mas podem não medir conjuntamente a auto-percepção das capacidades de inteligência emocional dos sujeitos. No entanto, de acordo com o método da correlação entre fatores utilizado em outros estudos, no presente estudo, verificou-se que todos os fatores se correlacionam significativamente, providenciando suporte para a sua convergência (Lahaye, Luminet, Van Broeck, Bodart, \& Mikolajczak, 2010). Deste modo, os estudos futuros deverão analisar estes resultados contraditórios, especificamente relacionados com os métodos de análise da validade convergente do QIE-AP.

O QIE-AP ao revelar propriedades psicométricas adequadas, parece cortar com a série negativa de instrumentos pouco confiáveis que têm vindo a ser desenvolvidos no âmbito da auto-percepção da IE (Mayer, 2006). As dificuldades de operacionalização do construto da IE têm vindo a ser as suas múltiplas definições conceptuais, a falta de convergência entre os seus modelos conceptuais, as dificuldades inerentes à sua medida, e por consequência, a questionabilidade da sua validade preditiva e incremental (Christiansen, Janovics, \& Siers, 2010; Landy, 2005; Mayer et al., 2008; Zeidner, Matthews, \& Roberts, 2004). Neste sentido, a existência de diversos modelos teóricos leva a uma falta de definição consensual sobre o construto da IE e das suas dimensões. Nesta perspetiva, Extremera-Pacheco e Fernández-Berrocal (2007) referem que se considerarmos a IE como uma capacidade mental composta por processos emocionais básicos, revela-se mais adequado utilizar as propostas de avaliação enumeradas por Mayer e Salovey (1997), quer sejam de auto-relato ou de performance. Se o investigador tem como objetivo avaliar habilidades intrapessoais, as medidas de auto-relato são uma opção bastante adequada. Isto porque ao confiar na introspeção permite avaliar processos emocionais subjacentes, dificilmente medidos através de tarefas de capacidade. Destaca-se assim que o QIE-AP é uma ferramenta que pretende ter capacidade preditiva, ao invés focar taxonomias de personalidade ou capacidades (Regner, 2008).

\section{Limitações e Investigação Futura}

Apesar dos resultados encorajadores dos dois estudos, devem ser apresentadas algumas limitações e delineadas direções futuras para a investigação. Em primeiro lugar, o presente estudo baseia-se em procedimentos de amostragem não probabilísticos onde constam, na sua maioria, jovens estudantes do ensino secundário. Neste sentido, a generalização dos resultados para outros grupos (e.g., idosos, populações com doença) considerando os fatores da IE, deverá ser foco de estudo de próximas investigações. Segundo, ambas as fases do presente estudo consideram exclusivamente métodos quantitativos de resposta aos itens. Talvez, a investigação futura poderá combinar outros métodos (e.g., entrevista) e relacionar outros instrumentos (e.g., EQ-I de Bar-On, 1997), com vista a avaliar a validade concorrente dos resultados do QIE-AP. Finalmente, ambas as fases são estudos transversais, focando-se num conjunto limitado de indicadores de auto-percepção de IE. Neste âmbito, os estudos futuros deverão considerar desenhos longitudinais que permitam avaliar a estabilidade e invariância temporal dos resultados 
do QIE-AP, relacionando-o com outras variáveis que suportem ainda mais a validade e confiabilidade do QIE-AP, na medida em que os dois estudos presentes sugerem que este instrumento é uma ferramenta promissora na área da auto-percepção da inteligência emocional.

\section{Referências}

Bar-On, R. (1997). Bar-On Emotional Quotient Inventory: A measure of emotional intelligence. Toronto, Canada: $\mathrm{O}$ Multi-Health Systems.

Bar-On, R. (2004). The Bar-On Emotional Quotient Inventory (EQ-i): Rationale, description, and summary of psychometric properties. In G. Geher (Ed.), Measuring emotional intelligence: Common ground and controversy (pp. 111-142). Hauppauge, NY: Nova Science.

Bedwell, S. (2003). Emotional intelligence, personality revisited or something else. Paper presented at the Symposium at the Annual Meeting of the Society of Industrial and Organizational Psychology, Orlando, FL, United States.

Bhullar, N., Schutte, N., \& Malouff, J. (2012). Trait emotional intelligence as a moderator of the relationship between psychological distress and satisfaction with life. Individual Differences Research, 10, 19-26.

Brackett, M., \& Mayer, J. (2003). Convergent, discriminant, and incremental validity of competing measures of emotional intelligence. Personality and Social Psychology Bulletin, 29, 1-12. doi:10.1177/0146167203254596

Brown, T. A. (2006). Confirmatory factor analysis for applied research. New York: Guilford.

Byrne, B. M. (2000). Structural equation modelling with AMOS: Basic concepts, applications, and programming. Mahwah, NJ: Lawrence Erlbaum.

Cheung, G. W., \& Rensvold, R. B. (2002). Evaluating goodness-of-fit indexes for testing measurement invariance. Structural Equation Modeling, 9, 233-255. doi:10.1207/ S15328007SEM0902_5

Christiansen, N. D., Janovics, J. E., \& Siers, B. P. (2010). Emotional intelligence in selection contexts: Measurement method, criterion-related validity, and vulnerability to response distortion. International Journal of Selection and Assessment, 18, 87-101. doi:10.1111/j.1468-2389.2010.00491.x

Extremera, N., \& Fernández-Berrocal, P. (2005). Perceived emotional intelligence and life satisfaction: Predictive and incremental validity using the Trait Meta-mood Scale. Personality and Individual Differences, 39, 937-948.

Extremera-Pacheco, N., \& Fernández-Berrocal, P. (2007). Una guía práctica de los instrumentos actuales de evaluación de la inteligencia emocional. In J. Navas \& P. Berrocal (Eds.), Manual de inteligencia emocional (pp. 99-122). Madrid, España: Pirámide.

Fornell, C., \& Larcker, D. F. (1981). Evaluating structural equation models with unobservable variables and measurement error. Journal of Marketing Research, 18, 39-50. doi: $10.2307 / 3151312$

Franco, M. (2003). A gestão das emoções na sala de aula: Projecto de modificação das atitudes de um grupo de docentes do $1^{\circ}$ ciclo do ensino básico. Lisboa, Portugal: Fundação Calouste Gulbenkian.

Franco, M. (2007). Inteligência emocional: Modelos, instrumentos de avaliação e limites. In A. Cadeias \& L. Almeida (Eds.), Inteligência humana: Investigação e aplicações: Vol. 1 (pp. 73-96). Coimbra, Portugal: Quarteto.
Gardner, H. (1995). Inteligências múltiplas: A teoria na prática. Porto Alegre, RS: Artmed. (Original publicado em 1993)

Goleman, D. (2003). Inteligência emocional. Lisboa, Portugal: Temas e Debates. (Original publicado em 1995)

Goldenberg, I., Matheson, K., \& Mantler, J. (2006). The assessment of emotional intelligence: A comparison of performance-based and self-report methodologies. Journal of Personality Assessment, 86, 33-45. doi:10.1207/ s15327752jpa8601_05

Hair, J. F., Black, B., Babin, B., \& Anderson, R. E. (2010). Multivariate data analysis ( $6^{\text {th }}$ ed.). Saddle River, NJ: Prentice Hall.

Hoyle, R. H., \& Smith, G. T. (1994). Formulating clinical research hypotheses as structural equation models: A conceptual overview. Journal of Consulting and Clinical Psychology, 62, 429-440. doi:10.1037/0022-006X.62.3.429

Kline, R. B. (2011). Beyond significance testing: Reforming data analysis methods in behavioral research. Washington, DC: American Psychological Association.

Lahaye, M., Luminet, O., Van Broeck, N., Bodart, E., \& Mikolajczak, M. (2010). Psychometric properties of the Emotion Awareness Questionnaire for children in a French-speaking population. Journal of Personality Assessment, 92, 317-326. doi:10.1080/00223891.2010.482003

Landy, F. J. (2005). Some historical and scientific issues related to research on emotional intelligence. Journal of Organizational Behaviour, 26, 411-424. doi:10.1002/job.317

Lima-Santos, N., \& Faria, L. (2005). Inteligência emocional: Adaptação do "Emotional Skills and Competence Questionnaire" (ESCQ) ao contexto português. Revista da Faculdade de Ciências Humanas e Sociais da Universidade Fernando Pessoa, 2, 275-289.

Limonero, J., Sábado, J., \& Fernández-Castro, J. (2006). Relación entre inteligencia emocional percibida y ansiedad ante la muerte en estudiantes universitario. Ansiedad e Estrés, 12, 267-278.

Loehlin, J. C. (2004). Latent variable models: An introduction to factor, path, and structural equation analysis ( $\left.4^{\text {th }} \mathrm{ed}.\right)$. Mahwah, NJ: Lawrence Erlbaum.

Mayer, J. (2006). A new field guide to emotional intelligence. In J. Ciarrochi, J. Forgas, \& J. Mayer (Eds.), Emotional intelligence in everyday life (pp. 3-6). Philadelphia, PA: Psychology Press.

Mayer, J., Caruso, D., \& Salovey, P. (2000). Emotional intelligence meets traditional standards for an intelligence. Intelligence, 27, 267-298. doi:10.1016/S0160-2896(99)00016-1

Mayer, J., \& Salovey, P. (1997). What is emotional intelligence? In P. Salovey \& D. Sluyter (Eds.), Emotional development and emotional intelligence (pp. 3-31). New York: Basic Books.

Mayer, J., Salovey, P., \& Caruso, D. (2002). Mayer-SaloveyCaruso Emotional Intelligence Test (MSCEIT) user's manual. Toronto, Canada: Multi-Health Systems.

Mayer, J., Salovey, P., Caruso, D., \& Sitarenios, G. (2003). Measuring emotional intelligence whit the MSCEIT V 2.0. Emotion, 3, 97-105. doi:10.1037/1528-3542.3.1.97

Mayer, J., Roberts, R., \& Barsade, S. (2008). Human abilities: Emotional intelligence. Annual Review Psychology, 59, 507536. doi:10.1146/annurev.psych.59.103006.093646

Nunnaly, J., \& Bernstein, I. (1994). Psychometric theory. New York: McGraw-Hill.

Petrides, K., \& Furnham, A. (2001). Trait emotional intelligence: Psychometric investigation with reference to established trait taxonomies. European Journal of Personality, 15, 425-448. doi:10.1002/per.416 
Teques, A. P., Llorca-Ramón, G., Bueno-Carrera, G., Pais-Ribeiro, J. \& Teques, P. (2015). Desenvolvimento e Avaliação das Características Psicométricas do Questionário de Auto-Percepção de Inteligência Emocional (QIE-AP).

Pérez, J. C., Petrides, K. V., \& Furnham, A. (2005). Measuring trait emotional intelligence. In R. Schulze \& R. D. Roberts (Eds.), International handbook of emotional intelligence ( $\mathrm{pp}$ 123-143). Cambridge, MA: Hogrefe \& Huber.

Pérez-González, J., Petrides, K., \& Furnham, A. (2007). La medida de la inteligencia emocional rasgo. In J. Navas \& P. Berrocal (Eds.), Manual de inteligencia emocional (pp. 8193). Madrid, España: Pirámide.

Queirós, M., Fernández-Berrocal, P., Extremera, N., Carral, J., \& Queirós, P. (2005). Validação e fiabilidade da versão portuguesa modificada da Trait Meta-Mood Scale. Psicologia, Educação e Cultura, 9, 199-216.

Regner, E. (2008). Validez convergente y discriminante del inventario de cociente emocional (EQ-I). Interdisciplinaria, 25(1), 29-51.

Roberts, R., Zeidner, M., \& Matthews, G. (2001). Does emotional intelligence meet traditional standards for an intelligence? Some new data and conclusions. Emotions, 1, 196-231. doi:10.1037/1528-3542.1.3.196

Rodrigues, N., Rebelo, T., \& Coelho, J. (2011). Adaptação da Escala de Inteligência Emocional de Wong e Law (WLEIS) e análise da sua estrutura factorial e fiabilidade numa amostra portuguesa. Psychologica, 55, 189-207.

Salovey, P., Mayer, D., Goldman, L., Turvey, C., \& Palfai, P. (1995). Emotional attention, clarity, and repair: Exploring emotional intelligence using the Trait Meta-Mood Scale. In J. Pennebaker (Ed.), Emotion, disclosure, \& Health (pp. 125151). Washington, DC: American Psychological Association.

Schutte, N., Malouff, J., \& Bhullar, N. (2007). The assessing emotions scale. In C. Stough, D. Saklofske, \& J. Parker (Eds.), The assessment of emotional intelligence. New York: Springer.

Schutte, N., Malouff, J., Hall, L., Haggerty, D., Cooper, J., \& Golden, C. (1998). Development and validation of a measure of emotional intelligence. Personality and Individual Differences, 25, 167-177. doi:10.1016/S0191-8869(98)00001-4

Schutte, N., Malouff, J., Simunek, M., McKenley, J., \& Hollander, S. (2002). Characteristic emotional intelligence and emotional well-being. Cognition and Emotion, 16, 769-785. doi:10.1080/02699930143000482

Schutte, N., Thorsteinsson, E., Donald, W., Hine, D. W., Foster, R., Cauchi, A., \& Binns, C. (2010). Experiential and rational processing styles, emotional intelligence and well-being. Australian Journal of Psychology, 62, 14-19. doi:10.1080/00049530903312865

Tabachnick, B. G., \& Fidell, L. S. (2007). Using multivariate statistics $\left(5^{\text {th }}\right.$ ed.). Boston, MA: Allyn \& Bacon.

Taksic, V., Takalcic, M., \& Brajkovic, S. (2001, June). Emotional intelligence: An empirical validation of the construct. Paper presented at the meeting of European Congress of Psychology, London, England.

Teques, A. (2009). Inteligencia emocional y acontecimientos de vida negativos (Tesis doctoral inédita, Universidad de Salamanca, España).

Veríssimo, R. (2005). Inteligência emocional, apoio social e regulação afectiva. Acta Médica Portuguesa, 18, 345-352.

Worthington, R. L, \& Whittaker, T. A. (2006). Scale development research: A content analysis and recommendations for best practices. The Counseling Psychologist, 34, 806-838. doi: $10.1177 / 0011000006288127$

Woyciekoski, C., \& Hutz, C. (2009). Inteligência emocional: Teoria, pesquisa, medida, aplicações e controvérsias Psicologia: Reflexão e Críticas, 22(1), 1-11. doi:10.1590/ S0102-79722009000100002
Zeidner, M., Matthews, G., \& Roberts, R. D. (2004). Emotional intelligence in the workplace: A critical review. Applied Psychology: An International Review, 53, 371-399. doi:10.1111/j.1464-0597.2004.00176.x

Zeidner, M., Shani-Zinovich, I., Matthews, G., \& Roberts, R. D. (2005). Assessing emotional intelligence in gifted and nongifted high school students: Outcomes depend on the measure. Intelligence, 33, 369-391. doi:10.1016/j.intell.2005.03.001
Recebido: 10/03/2014

$1^{a}$ revisão: $10 / 04 / 2014$

Aceite final: 16/04/2014 